\title{
Metabolomics for predicting hyperglycemia in pregnancy: a protocol for a systematic review and potential meta-analysis
}

\author{
Bianca Fioravanti Nicolosi ${ }^{1}$, Debora F. Leite ${ }^{2}$, Jussara Mayrink², Renato T. Souza ${ }^{2}$, José Guilherme Cecatti ${ }^{2^{*}}$ and
} Iracema de Mattos Paranhos Calderon ${ }^{1}$

\begin{abstract}
Background: Hyperglycemia in pregnancy (HIP) has been recently differentiated between diabetes in pregnancy (DIP) and gestational diabetes mellitus (GDM). The proposed protocol is relevant, and clinical concern is due to the higher risk of adverse pregnancy outcomes (APO) and long-term effects on both the mother and the fetus. Fasting plasma glucose level (FPG) and oral glucose tolerance test (OGT) are current diagnostic tools. However, controversy persists concerning diagnostic criteria, cut-off points, and even selective or universal screening. The objective of this systematic review is to assess the performance of metabolomic markers in the prediction of HIP.

Methods: This is a protocol for a systematic review with potential meta-analysis. The primary outcome is GDM, defined as glucose intolerance identified in the second and third trimesters of pregnancy (any FPG $\geq 92 \mathrm{mg} / \mathrm{dL}$ and $<126 \mathrm{mg} / \mathrm{dL}$ OR when 75-g OGTT shows one altered value among these: FPG $\geq 92 \mathrm{mg} / \mathrm{dL}$ or 1-h post glucose load $\geq 180 \mathrm{mg} / \mathrm{dL}$ or 2 -h post glucose load $\geq 153 \mathrm{mg} / \mathrm{dL}$ ); the secondary outcome is HIP, defined as hyperglycemia detected in the first trimester of pregnancy (any FPG $\geq 126 \mathrm{mg} / \mathrm{dL}$ ). A detailed systematic literature search will be carried out in electronic databases and conference abstracts, using the keywords "gestational diabetes mellitus," "metabolomics," "pregnancy," and "screening" (and their variations). We will include original peer-reviewed articles published from Jan 1, 1999, to Dec 31, 2018. Original studies including diabetes diagnosed before pregnancy (T2DM and T1DM), multiple pregnancies, and congenital malformations will be excluded. All results regarding samples, participant characteristics, metabolomic techniques, and diagnostic accuracy measures will be retrieved and analyzed. Since this is a systematic review, no ethical approval is necessary.
\end{abstract}

Discussion: This systematic review may have the potential to provide significant evidence-based findings on the prediction performance of metabolomics. There are short and long-term repercussions for the mother and the newborn. Therefore, both may benefit from an accurate prediction technique for HIP.

Systematic review registration: This protocol was registered in the PROSPERO platform under number CRD42018100175.

Keywords: Gestational diabetes mellitus, Hyperglycemia in pregnancy, High-risk pregnancy, Metabolomics, biomarkers

\footnotetext{
* Correspondence: cecatti@unicamp.br

${ }^{2}$ Department of Obstetrics and Gynaecology, School of Medicine, University

of Campinas (UNICAMP), Campinas, São Paulo, Brazil

Full list of author information is available at the end of the article
}

(c) The Author(s). 2019 Open Access This article is distributed under the terms of the Creative Commons Attribution 4.0 International License (http://creativecommons.org/licenses/by/4.0/), which permits unrestricted use, distribution, and reproduction in any medium, provided you give appropriate credit to the original author(s) and the source, provide a link to the Creative Commons license, and indicate if changes were made. The Creative Commons Public Domain Dedication waiver (http://creativecommons.org/publicdomain/zero/1.0/) applies to the data made available in this article, unless otherwise stated. 


\section{Background}

The current classification of hyperglycemia in pregnancy (HIP) distinguishes diabetes mellitus in pregnancy (DIP), diagnosed before 20 weeks, from gestational diabetes mellitus (GDM), identified in the second or third trimester of gestation [1, 2]. When criteria for GDM are not fully met, less severe degrees of GDM also produce adverse effects on the mother and newborn [3-5]. Nevertheless, women with mild gestational hyperglycemia (MGH) - those with hyperglycemia in the glycemic profile, despite a normal response to oral glucose tolerance test (OGTT) - have been identified in specialized obstetrical centers [6, 7]. This subgroup of women is at higher risk of maternal and perinatal adverse outcomes, suggesting that their glycemic levels should also be controlled during pregnancy. More recently, despite several new criteria and cut-off points proposed for the diagnosis of GDM $[1,8]$, it is estimated that MGH women account for more than $15 \%$ of cases. Therefore, women with MGH are more likely to be identified and treated as those with GDM [9].

Universal screening for gestational diabetes is initially based on a fasting plasma glucose (FPG) measurement during the first prenatal visit (preferably before 20 weeks). DIP is diagnosed when FPG $\geq 126 \mathrm{mg} / \mathrm{dL}$ or GDM is considered when FPG is $\geq 92 \mathrm{mg} / \mathrm{dL}$ and $<126$ $\mathrm{mg} / \mathrm{dL}$. An OGTT of $75 \mathrm{~g}$ is recommended between 24 and 28 weeks of gestation, when initial FPG $<92 \mathrm{mg} / \mathrm{dL}$, and GDM is considered when FPG $\geq 92 \mathrm{mg} / \mathrm{dL}$ or 1 - $\mathrm{h}$ post glucose load $\geq 180 \mathrm{mg} / \mathrm{dL}$ or 2 -h post glucose load $\geq 153 \mathrm{mg} / \mathrm{dL}[1,8]$. This recommendation is still controversial due to the high costs involved and the lack of long-term evidence of any benefit $[2,10]$. In this context, race/ethnicity, family history, body mass index (BMI), and prior history of GDM have been tested as the first screening tools for GDM in prediction models. However, their modest results may be due to low prevalence rates (reaching a maximum of $50 \%$ in pregnant women with GDM) and insufficient accuracy. This might lead to diagnostic testing in many women who would not require testing, particularly during late pregnancy [11-14].

The earlier the diagnosis and treatment, the better the prognosis of HIP. Thus, early identification of women at risk is critical. Research efforts have targeted first-trimester data collection. Many types of biomarkers have been tested, alone or in prediction models. Lower levels of adiponectin, sex-hormone-binding globulin, and high levels of C-reactive protein have been associated with an increased risk for GDM [15-17]. Likewise, studies have provided insight into the prediction of diabetes using metabolites of different chemical classes, resulting in diverse prediction performances. The advantage of metabolomics is that it has the potential to capture disease-relevant metabolic changes and identify novel biomarkers of disease processes [18].
Metabolomic studies in GDM have addressed biomarkers for early risk stratification, therapeutic effects of insulin and dietary treatment, and the risk of transition from GDM to short- or long-term T2DM. Several studies have identified some metabolites for GDM such as amino acids and their derivatives, organic acids, lipids, and fatty acids. However, they are extremely diverse in several aspects: (i) clinical controversies in GDM diagnostic protocol, (ii) gestational age for participant inclusion, (iii) biological samples (blood, urine) used, and (iv) instrumental platforms employed- mass spectrometry (MS), liquid chromatography (LC), gas chromatography (GC) or capillary electrophoresis $(\mathrm{CE})$, or proton nuclear magnetic resonance ( $\left.{ }^{1} \mathrm{H}-\mathrm{NMR}\right)$ spectroscopy [19-24]. These variables may explain the inconsistent findings [25].

This scenario and the lack of a systematic review (SR) on metabolomic markers as risk predictors to HIP (DIP or GDM) justify the proposition of this protocol. The source or type of the samples evaluated and the different analytical techniques will be evaluated; the results will help define the role of biomarkers in the identification of women at risk of hyperglycemia during pregnancy. Thus, the objective of the proposed SR is to assess the performance of metabolomic markers in pregnant women to predict HIP.

\section{Methods/design \\ Review question}

As a result of the social and economic implications of HIP disorders, their consequences to maternal and fetal lives worldwide, in addition to the increasing applicability of omics technologies, this systematic review will be guided by the following research question: "What is the performance of metabolomic markers in pregnant women to predict HIP, independently of DIP or GDM?"

\section{Condition or domain studied}

Pregnant women with hyperglycemia in pregnancy (HIP)_categorized as gestational diabetes mellitus (GDM) or diabetes in pregnancy (DIP) $[1,2]$-is the condition to be assessed.

\section{Participants/population}

We will include original studies involving pregnant women with hyperglycemia, irrespective of the diagnostic criteria for this condition.

\section{Interventions/exposure}

The intervention to be analyzed is screening for HIP (GDM or DIP) with the metabolomic approach. Maternal samples need to be drawn earlier than standardized recommendations for hyperglycemia screening in pregnancy. 


\section{Study inclusion and exclusion criteria}

Inclusion criteria: Original cohort or case-control studies including women who underwent any screening test for hyperglycemia or GDM during pregnancy.

Exclusion criteria: Studies on pregnant women with previous diabetes mellitus, either type 1 or type 2, diagnosed before metabolomic screening during pregnancy. In addition, all studies including pregnant women under health conditions that can affect, directly or indirectly, the levels of glycemia across pregnancy, that is, pancreas or thyroid cancer, severe obesity, chronic use of corticosteroids, and others, will also be excluded.

\section{Outcomes}

The primary outcomes will be the diagnosis of GDM or hyperglycemia, regardless of the diagnostic criteria applied.

\section{Literature search}

The primary source of information will be the electronic databases: PubMed, EMBASE, LILACS, SciELO, CINAHL, MIDIRS, Scopus, and Web of Science. Secondary sources will include Google Scholar, handsearching the reference list for eligible studies, conference proceedings, and direct contact with authors, when necessary. In addition, the reference's list of each selected article will be checked for finding some study not yet identified with the previous procedures.

The original search strategy proposed is the follows: “\#1 "Metabolomics"[Mesh] OR Metabolomic OR Metabonomics OR Metabonomic; \#2 "Diabetes, Gestational"[Mesh] OR Diabetes, Pregnancy-Induced OR Diabetes, Pregnancy Induced OR Pregnancy-Induced Diabetes OR Gestational Diabetes OR Diabetes Mellitus, Gestational OR Gestational Diabetes Mellitus; \#3 "Pregnancy in Diabetics" [Mesh] OR Pregnancy in Diabetic OR Pregnancy in Diabetes OR Pregnancy in Diabete; \#4 "Hyperglycemia"[Mesh] OR Hyperglycemias OR Hyperglycemia, Postprandial OR Hyperglycemias, Postprandial OR Postprandial Hyperglycemias OR Postprandial Hyperglycemia; \#5 "Pregnancy"[Mesh] OR Pregnancies OR Gestation; \#1 AND \#2 OR \#3 OR (\#4 AND \#5)".

The keywords linked to the outcomes of interest (hyperglycemia OR gestational diabetes screening) will be combined with terms related to metabolomic (markers, samples, and technique) and pregnancy, using Boolean connectors and specific Mesh. The same search strategy will be applied for each database, adapting for individual filters, main language, their own syntax, and mechanisms of search. The complete search strategy is provided as Additional file 1.

Studies published in the last 20 years (from Jan 1, 1999, to Dec 31, 2018) will be taken into account. The search strategy will be re-run before final analysis, to check for recently published eligible studies. There will be no language restrictions; selected studies in a language different from English, French, Spanish, and Portuguese will be translated and considered for eligibility.

\section{Data extraction and management}

Literature search, study selection, and data extraction will be performed independently by two investigators (BFN, DFBL). Firstly, titles and abstracts will be assessed, and when more data is needed, the full texts will be read to decide on study inclusion. All disagreements will be discussed until a consensus is reached, and when necessary, with the collaboration of a third senior investigator. Academic supervisors (IMPC, JGC) will resolve any discrepancies and will be responsible for checking and approving each step of the review.

Variables to be extracted include authors and year of publication, study design, definition or protocol used for GDM/Hyperglycemia diagnosis, number of participants enrolled (pregnant women screened with and without GDM/Hyperglycemia), gestational age of assessment (both for metabolomics and for standardized GDM diagnosis), laboratory methods applied (e.g., technique, year of sample collection, year of laboratory analysis), and biological sample analyzed (e.g., blood, amniotic fluid). Authors will be contacted in case any additional data is required. The Kappa index will be used to evaluate the degree of agreement in the selection and data extraction performed by two different investigators (BFN, DFBL).

\section{Risk of bias assessment}

Both investigators initially involved with the literature search (BFN and DFBL) will assess the methodological quality of all included studies. They must check their criteria and discuss any discordance risk of bias. A third investigator should resolve any disagreement if necessary, and final decisions should be made by the majority. The "Quality Assessment of Diagnostic Accuracy Studies" (QUADAS-2) tool will be used [26]. This tool comprises 4 domains: patient selection, index test, reference standard, and flow and timing. Each domain is assessed in terms of risk of bias, and the first 3 domains are also assessed in terms of concerns regarding applicability. Signaling questions are included to help judge the risk of bias. The QUADAS-2 tool is applied in 4 phases: summarize the review question, tailor the tool and produce review-specific guidance, construct a flow diagram for the primary study, and judge bias and applicability. This tool will allow for a more transparent rating of bias and applicability of primary diagnostic accuracy studies. 


\section{Strategy for data synthesis}

An aggregate pregnant women data synthesis will be performed with all included studies. Narrative data will be analyzed and structured according to the results of diagnostic protocols applied. If possible, a meta-analysis (hierarchical summary of the receiver characteristic operating curve) of diagnostic accuracy measures will be conducted. For summarizing the effects in case a metaanalysis is feasible, a fixed effects model will preferably be employed if heterogeneity is judged to be low enough; otherwise, a random effects model will be used. If available data does not allow a quantitative synthesis, a qualitative descriptive approach will be used instead.

Regarding the Preferred Reporting Items for Systematic Reviews and Meta-Analysis (PRISMA) statement recommendations, a flow diagram will show details of the literature search and study selection [27]. Characteristics of the included studies and the risk of bias assessment will be shown in tables. Depending on data availability, we will perform subgroup analysis according to blood glucose levels (non-diabetic, hyperglycemia in pregnancy, and gestational diabetes mellitus).

\section{Potential limitations of this review}

Metabolomics is a novel technology that does not have a unique and complete definition of methods, analytical platforms, and specimens to improve accuracy. Likewise, hyperglycemia in pregnancy has conflicting diagnostic criteria, cut-off points, and screening tests, in addition to an important gap in long-term maternal and fetal adverse outcomes. These issues, in conjunction with marked heterogeneity of the included population, may influence study quality, and thus the expected results.

\section{Ethics and dissemination}

Since this is a systematic review protocol, no ethics committee approval is necessary. This protocol was registered in the International Prospective Register of Systematic Reviews (PROSPERO) database, number CRD42018100175, and adheres to the Preferred Reporting Items for Systematic Reviews and Meta-Analysis Protocols (PRISMA-P) statement [27].

Our research group is committed to the advance in the metabolomic field of reproductive health, performing systematic reviews (CRD42018089985; CRD42018097409; CRD42018100172) about metabolomic markers as predictive tools on adverse pregnancy outcomes. Therefore, this protocol will be electronically available at our website (http://www.medscinet.com/samba), and a report will be sent to our sponsors. The authors will submit the systematic review results for publication in peer-reviewed scientific journals.

\section{Patient and public involvement}

There was no patient or public involvement in this systematic review protocol.

\section{Discussion}

In general, GDM is only identified and treated at the end of the second trimester of pregnancy, after clinical diagnosis. Early identification of women at risk for GDM has been a major challenge because the ideal tool for this was not yet defined. Likewise, significant gaps still remain in the understanding of genetic and environmental risk factors, as well as related mechanisms that contribute to GDM. According to some authors, metabolome studies provide a general summary of metabolic interactions within a given biological system and allow the simultaneous identification and quantification of a large number of analytes in a highly productive and unbiased manner [28]. In this context, metabolomics emerged as a technology with a potential for the early detection of GDM, besides to favor the understanding of the pathogenesis and the impact of the disease in the mother and her offspring [29].

Our study intends to identify metabolomic markers that will potentially clarify some gaps in HIP (GDM or DIP), considering the different criteria for GDM/hyperglycemia in pregnancy and metabolomic techniques. Thus, a standardized and detailed protocol for a systematic review of early metabolomic predictors is of great importance to ensure significant evidence-based findings on the prediction performance of metabolomics. There are short- and long-term repercussions for the mother and the newborn, and both might benefit from an accurate prediction technique for HIP. Furthermore, the results of this systematic review may suggest new studies on the topic, focused on identifying the best technique for metabolomic screening.

\section{Additional file}

Additional file 1: Metabolomics for predicting gestational diabetes

mellitus: protocol for a systematic review and meta-analysis. (DOC $60 \mathrm{~kb}$ )

\section{Abbreviations \\ ${ }^{1} \mathrm{H}$-NMR: Proton nuclear magnetic resonance spectroscopy; APO: Adverse pregnancy outcomes; BMI: Body mass index; CE: Capillary electrophoresis; DIP: Diabetes in pregnancy; FPG: Fasting plasma glucose levels; GC: Gas chromatography; GDM: Gestational diabetes mellitus; HIP: Hyperglycemia in pregnancy; LC: Liquid chromatography; MGH: Mild gestational hyperglycemia; MS: Mass spectrometry; OGTT: Oral glucose tolerance test; T1DM: Type 1 diabetes mellitus; T2DM: Type 2 diabetes mellitus}

\section{Acknowledgements}

We would like to thank the librarians from the Unesp Library, Botucatu Medical School, São Paulo State University, Brazil, and also those from the Unicamp Library, School of Medicine, Brazil, for their assistance with the literature search strategy. 


\section{Authors' contributions}

BFNG and DFBL developed the systematic review protocol and will perform the literature search, study selection, data extraction, and risk of bias assessment. RTS, JM, JGC, and IMPC supervised the protocol elaboration and will later resolve any discrepancies in methodology. BFNG, DFBL, and RTS will deal with statistical procedures. JGC and IMPC performed the last amendments and will monitor the development of the protocol until approval of the final version of the manuscript. All authors read the systematic review protocol and agreed with its submission.

\section{Funding}

This research was supported by the Brazilian National Research Council (CNPQ grant number 401636/2013-5) and Bill and Melinda Gates Foundation (grant number OPP1107597- Grand Challenges Brazil: Reducing the burden of preterm birth), which provided funding for the PRETERM-SAMBA project (www.medscinet.com/samba). They played no role in the development of the proposal.

\section{Availability of data and materials}

This is a protocol for a systematic review, and therefore, no data are generated or analyzed. After completion, all search strategies, results, and compiled information will be included in the corresponding article to be published and stored in potential Supplementary Information files.

\section{Ethics approval and consent to participate}

Since this is a systematic review protocol, no ethics committee approval is necessary. This protocol was registered in the International Prospective Register of Systematic Reviews (PROSPERO) database, under number CRD42018100175

\section{Consent for publication}

Not applicable.

\section{Competing interests}

IMPC gives speeches in conferences and publishes articles on gestational diabetes mellitus. BFN, DFBL, RTS, JM, JGC, and IMPC are developing original studies on metabolomics and pregnancy-related disorders, including gestational diabetes mellitus.

\section{Author details}

'Department of Gynaecology and Obstetrics, Botucatu Medical School, São Paulo State University-UNESP, Botucatu, São Paulo, Brazil. ${ }^{2}$ Department of Obstetrics and Gynaecology, School of Medicine, University of Campinas (UNICAMP), Campinas, São Paulo, Brazil.

Received: 27 November 2018 Accepted: 13 August 2019 Published online: 24 August 2019

\section{References}

1. World Health Organization. Diagnostic criteria and classification of hyperglycaemia first detected in pregnancy. Geneva: WHO; 2013. [http:// apps.who.int/iris/bitstream/handle/10665/85975/who_nmh_mnd_13.2_eng. pdf;jsessionid=329671853FD2D7858609593D67A08333? sequence=1]

2. Hod M, Kapur A, Sacks DA, Hadar E, Agarwal M, Di Renzo GC, Cabero Roura $L$, Mclntyre HD, Morris $J$, Divakar H. The International Federation of Gynecology and Obstetrics (FIGO) Initiative on gestational diabetes mellitus: a pragmatic guide for diagnosis, management, and care. Int J Gynaecol Obstet. 2015;131(Suppl 3):S173-211.

3. Rudge MV, Calderon IM, Ramos MD, Brasil MA, Rugolo LM, Bossolan G, Odland JO. Maternal daily hyperglycemia diagnosed by glycemic profile: a maternal and perinatal public health problem. Rev Bras Ginecol Obstet. 2005;27(11):691-7.

4. Landon MB, Spong CY, Thom E, Carpenter MW, Ramin SM, Casey B, Wapner RJ, Varner MW, Rouse DJ, Thorp JM Jr, Sciscione A, Catalano P, Harper M, Saade G, Lain KY, Sorokin Y, Peaceman AM, Tolosa JE, Anderson GB. Eunice Kennedy Shriver National Institute of Child Health and Human Development Maternal-Fetal Medicine Units Network. A multicenter, randomized trial of treatment for mild gestational diabetes. N Engl J Med. 2009;361(14):1339-48.

5. Landon MB, Rice MM, Varner MW, Casey BM, Reddy UM, Wapner RJ, Rouse DJ, Biggio JR Jr, Thorp JM, Chien EK, Saade G, Peaceman AM, Blackwell SC,
Van Dorsten JP. Eunice Kennedy Shriver National Institute of Child Health and Human Development Maternal-Fetal Medicine Units (MFMU) Network. Mild gestational diabetes mellitus and long-term child health. Diabetes Care. 2015:38(3):445-52.

6. Rudge MV, Peraçoli JC, Berezowski AT, Calderon IM, Brasil MA. The oral glucose tolerance test is a poor predictor of hyperglycemia during pregnancy. Braz J Med Biol Res. 1990;23(11):1079-89.

7. Moreli JB, Santos JH, Lorenzon-Ojea AR, Corrêa-Silva S, Fortunato RS, Rocha CR, Rudge MV, Damasceno DC, Bevilacqua E, Calderon IM. Hyperglycemia differentially affects maternal and fetal DNA integrity and DNA damage response. Int J Biol Sci. 2016;12(4):466-77.

8. American Diabetes Association. Classification and diagnosis of diabetes. Diabetes Care. 2017:40(Suppl. 1):S11-24.

9. Sirimarco MP, Guerra HM, Lisboa EG, Vernini JM, Cassetari BN, Costa RA, Rudge MV, Calderon IM. Diagnostic protocol for gestational diabetes mellitus (GDM) (IADPSG/ADA, 2011): influence on the occurrence of GDM and mild gestational hyperglycemia $(\mathrm{MGH})$ and on the perinatal outcomes. Diabetol Metab Syndr. 2017;9:2

10. Sacks DB, Coustan DR, Cundy T, Donovan L, Hod M. Gestational diabetes mellitus: why the controversy? Clin Chem. 2018;64(3):431-8.

11. Van Leeuwen $M$, Opmeer BC, Zweers EJ, van Ballegooie E, ter Brugge HG, de Valk HW, Visser GH, Mol BW. Estimating the risk of gestational diabetes mellitus: a clinical prediction model based on patient characteristics and medical history. BJOG. 2010;117(1):69-75.

12. Cosson E, Benbara A, Pharisien I, Nguyen M, Revaux A, Lormeau B, SandreBanon D, Assad N, Pillegand C, Valensi P, Carbillon L. Diagnostic and prognostic performances over 9 years of a selective screening strategy for gestational diabetes mellitus in a cohort of 18,775 subjects. Diabetes Care. 2012;36(3):598-603.

13. Zhang C, Rawal S, Chong Y. Risk factors for gestational diabetes: is prevention possible? Diabetologia. 2016;59(7):1385-90.

14. Cosson E, Carbillon L, Valensi P. High fasting plasma glucose during early pregnancy: a review about early gestational diabetes mellitus. J Diabetes Res. 2017;2017:8921712

15. Wolf M, Sandler L, Hsu K, Vossen-Smirnakis K, Ecker JL, Thadhani R. Firsttrimester $\mathrm{C}$-reactive protein and subsequent gestational diabetes. Diabetes Care. 2003;26:819-24.

16. Smirnakis KV, Plati A, Wolf M, Thadhani R, Ecker JL. Predicting gestational diabetes: choosing the optimal early serum marker. Am J Obstet Gynecol. 2007;196(410):e411-6.

17. Savvidou M, Nelson SM, Makgoba M, Messow CM, Sattar N, Nicolaides K. First-trimester prediction of gestational diabetes mellitus: examining the potential of combining ma- ternal characteristics and laboratory measures. Diabetes. 2010:59:3017-22.

18. Connor SC, Hansen MK, Corner A, Smith RF, Ryan TE. Integration of metabolomics and transcriptomics data to aid biomarker discovery in type 2 diabetes. Mol BioSyst. 2010;6(5):909-21.

19. Liu T, Li J, Xu F, Wang M, Ding S, Xu H, Dong F. Comprehensive analysis of serum metabolites in gestational diabetes mellitus by UPLC/Q-TOF-MS. Anal Bioanal Chem. 2016;408(4):1125-35.

20. Chorell E, Hall UA, Gustavsson C, Berntorp K, Puhkala J, Luoto R, Olsson T, Holmäng A. Pregnancy to postpartum transition of serum metabolites in women with gestational diabetes. Metabolism. 2017;72:27-36.

21. Bentley-Lewis R, Xiong G, Lee H, Yang A, Huynh J, Kim C. Metabolomic analysis reveals amino-acid responses to an oral glucose tolerance test in women with prior history of gestational diabetes mellitus. J Clin Transl Endocrinol. 2014;1(2):38-43.

22. Dudzik D, Zorawski M, Skotnicki M, Zarzycki W, García A, Angulo S, Lorenzo MP, Barbas C, Ramos MP. GC-MS based gestational diabetes mellitus longitudinal study: identification of 2-and 3- hydroxybutyrate as potential prognostic biomarkers. J Pharm Biomed Anal. 2017;144:90-8.

23. Lappas M, Mundra PA, Wong G, Huynh K, Jinks D, Georgiou HM, Permezel $\mathrm{M}$, Meikle PJ. The prediction of type 2 diabetes in women with previous gestational diabetes mellitus using lipidomics. Diabetologia. 2015;58(7):1436-42.

24. Allalou A, Nalla A, Prentice KJ, Liu Y, Zhang M, Dai FF, Ning JX, Osborne LR, Cox BJ, Gunderson EP, Wheeler MB. A predictive metabolic signature for the transition from gestational diabetes mellitus to type 2 diabetes. Diabetes. 2016;65(9):2529-39.

25. Mao X, Chen X, Chen C, Zhang H, Law KP. Metabolomics in gestational diabetes. Clin Chim Acta. 2017:475:116-27. 
26. Whiting PF, Rutjes AW, Westwood ME, Mallett S, Deeks JJ, Reitsma JB, Leeflang MM, Sterne JA, Bossuyt PM. QUADAS- a revised tool for the quality assessment of diagnostic accuracy studies. Annals Intern Med. 2011;155(8):529-36.

27. Moher D, Shamseer L, Clarke M, Ghersi D, Liberati A, Petticrew M, Shekelle P, Stewart LA, PRISMA-P Group. Preferred reporting items for systematic review and meta-analysis protocols (PRISMA-P) 2015 statement. Syst Rev. 2015;4:1.

28. Tokarz J, Haid M, Cecil A, Prehn C, Artati A, Moller G, Adamski J. Endocrinology meets metabolomics: achievements, pitfalls, and challenges. Trends Endocrinol Metab. 2017;28:705-21.

29. O'Neill K, Alexander J, Azuma R, Xiao R, Snyder NW, Mesaros CA, Blair IA, Pinney SE. Gestational Diabetes Alters the Metabolomic Profile in 2nd Trimester Amniotic Fluid in a Sex-Specific Manner. Int J Mol Sci. 2018;19(9). https://doi.org/10.3390/ijms19092696.

\section{Publisher's Note}

Springer Nature remains neutral with regard to jurisdictional claims in published maps and institutional affiliations.

Ready to submit your research? Choose BMC and benefit from:

- fast, convenient online submission

- thorough peer review by experienced researchers in your field

- rapid publication on acceptance

- support for research data, including large and complex data types

- gold Open Access which fosters wider collaboration and increased citations

- maximum visibility for your research: over $100 \mathrm{M}$ website views per year

At $B M C$, research is always in progress.

Learn more biomedcentral.com/submissions 